Supporting information for:

Sulfur Radical Cations. Kinetic and Products Study of the Photo-Induced Fragmentation Reactions of (Phenylsulfanylalkyl)trimethylsilanes and Phenylsulfanylacetic Acids Radical Cations.

Enrico Baciocchi, * Tiziana Del Giacco, * Fausto Elisei, Andrea Lapi,

Table of contents:

Preparation and characterization of $\mathbf{2}$ and $\mathbf{4 .}$

Table S1. Absorption maxima and transients produced by LFP of $\mathrm{NMQ}^{+} /$toluene/PhSCH(R)X in $\mathrm{N}_{2}$-saturated $\mathrm{CH}_{3} \mathrm{CN}$.

Figure S1. Observed rate constant ( $\left.k_{\mathrm{obs}}\right)$ vs [4-CN-pyridine] and $1 / k_{\mathrm{obs}} v s 1 /[4-\mathrm{CN}$-pyridine] for the decay of $\mathbf{3}^{+\bullet}$ in $\mathrm{CH}_{3} \mathrm{CN}$.

Figure S2. Time-resolved absorption spectra of the 1,4-dicyanonaphthalene/ $\mathrm{PhSCH}(\mathrm{Ph}) \mathrm{CO}_{2} \mathrm{NMe}_{4}$ system in $\mathrm{N}_{2}$-saturated $\mathrm{CH}_{3} \mathrm{CN}$. 


\section{Preparation and characterization of 2 and 4.}

Phenyl(phenylsulfanyl)acetic acid (4). ${ }^{\mathrm{S} 1}$ Butyllithium, (1.6 M in hexane, $6.4 \mathrm{~mL}, 10 \mathrm{mmol}$ ) was slowly added in a $50 \mathrm{~mL}$ three necked round bottom flask containing a stirred solution of benzyl phenyl sulfide $(2 \mathrm{~g}, 10 \mathrm{mmol})$ in $30 \mathrm{~mL}$ of anhydrous THF at $0{ }^{\circ} \mathrm{C}$ under Ar atmosphere. The mixture was allowed to react for $1 \mathrm{~h}$ after which a slurry of diethyl ether and crunched solid $\mathrm{CO}_{2}$ was slowly added. After the removal of $\mathrm{CO}_{2}$ water was added and the aqueous layer was separated, filtered and acidified with diluted $\mathrm{HCl}$. The obtained white solid was then filtrated, treated with dilute aqueous $\mathrm{Na}_{2} \mathrm{CO}_{3}$. Filtration and acidification of the aqueous solution gave $1.7 \mathrm{~g}$ (70\% yield) of a white solid. m. p. 101$103{ }^{\circ} \mathrm{C}$ (lit. $\left.102-103^{\circ} \mathrm{C}\right)^{\mathrm{S} 1}$

${ }^{1} \mathrm{H}-\mathrm{NMR}\left(300 \mathrm{MHz}, \mathrm{CDCl}_{3}\right): \delta:$ 7.2-7.5 (m, $\left.10 \mathrm{H}\right), 4.9(\mathrm{~s}, 1 \mathrm{H}) .{ }^{\mathrm{S} 2}$

[Phenyl(phenylsulfanyl)methyl] trimethylsilane (2). ${ }^{\mathrm{S} 3}$ Butyllithium, (1.6 $\mathrm{M}$ in hexane, $6.4 \mathrm{~mL}, 10$ mmol) was slowly added in a $50 \mathrm{~mL}$ three necked round bottom flask containing a stirred solution of benzyl phenyl sulfide $(2 \mathrm{~g}, 10 \mathrm{mmol})$ in $30 \mathrm{~mL}$ of anhydrous THF at $0{ }^{\circ} \mathrm{C}$ under $\mathrm{Ar}$ atmosphere. The mixture was allowed to react for $1 \mathrm{~h}$ after which trimethylsilyl chloride $(1.4 \mathrm{~mL}, 10 \mathrm{mmol})$ was slowly added. The mixture was stirred $30 \mathrm{~min}$ at room temperature, poured in a saturated $\mathrm{NH}_{4} \mathrm{Cl}$ aqueous solution $(40 \mathrm{~mL})$ and extracted three times with diethyl ether. The recombined organic layers were dried over anhydrous $\mathrm{Na}_{2} \mathrm{SO}_{4}$ and evaporated. After silica gel chromatography purification (eluant: hexane/ethyl acetate 9:1) $2.6 \mathrm{~g}$ of a colorless oil were obtained (96\% yield).

${ }^{1} \mathrm{H}-\mathrm{NMR}\left(300 \mathrm{MHz}, \mathrm{CDCl}_{3}\right):$ : $: 7.0-7.4(\mathrm{~m}, 10 \mathrm{H}), 3.8(\mathrm{~s}, 1 \mathrm{H}), 0.1(\mathrm{~s}, 9 \mathrm{H}) .{ }^{\mathrm{S} 3}$

EI-MS (70 V): 73(100\%) [ $\left.{ }^{+} \mathrm{SiMe}_{3}\right], 272(50 \%)\left[\mathrm{M}^{+}\right], 199(23 \%), 195(27 \%), 167$ (27\%), 135 (28\%), $122(22 \%)$.

(S1) Lehto, S.; Shirley, D. A. J. Org. Chem. 1957, 22, 989.

(S2) Ogura, K.; Itoh, H.; Morita, T.; Sanada, K.;Iida, H. Bull. Chem. Soc. Jpn. 1982, 55, 1216.

(S2) Ager, D. J. J. Chem. Soc., Perkin Trans. I 1986, 195. 
Table S1. Absorption maxima and transients produced by LFP of $\mathrm{NMQ}^{+} /$toluene/sulfide in $\mathrm{N}_{2}$-saturated $\mathrm{CH}_{3} \mathrm{CN}$.

\begin{tabular}{|c|c|c|c|c|}
\hline compd & $\mathrm{R}$ & $X$ & $\lambda_{\max }(\mathrm{nm})$ & transient \\
\hline \multirow[t]{5}{*}{1} & $\mathrm{H}$ & $\mathrm{Si}\left(\mathrm{CH}_{3}\right)_{3}$ & $330-340$ & $1^{+\bullet} ; \mathrm{C}_{6} \mathrm{H}_{5} \mathrm{SCH}_{2}^{\bullet}$ \\
\hline & & & 400 & NMQ• \\
\hline & & & $540-560$ & $1^{+\bullet}, \mathrm{NMQ}^{\bullet}$ \\
\hline & & & $>700$ & $1_{2}^{+\bullet}$ \\
\hline & & & $330,550^{\mathrm{a}}$ & $1^{+\bullet}$ \\
\hline \multirow[t]{5}{*}{2} & $\mathrm{C}_{6} \mathrm{H}_{5}$ & $\mathrm{Si}\left(\mathrm{CH}_{3}\right)_{3}$ & $330-360$ & $2^{+\bullet} ; \mathrm{C}_{6} \mathrm{H}_{5} \mathrm{SC}\left(\mathrm{C}_{6} \mathrm{H}_{5}\right) \mathrm{H}^{\bullet}$ \\
\hline & & & 400 & $\mathrm{NMQ}^{\bullet}$ \\
\hline & & & 540 & $2^{+\bullet}, \mathrm{NMQ}^{\bullet}$ \\
\hline & & & $>700$ & $22^{+\bullet}$ \\
\hline & & & $<350,530^{\mathrm{a}}$ & $2^{+\bullet}$ \\
\hline \multirow[t]{5}{*}{3} & $\mathrm{H}$ & $\mathrm{COOH}$ & $325-340$ & $3^{+\bullet} ; \mathrm{C}_{6} \mathrm{H}_{5} \mathrm{SCH}_{2}^{\bullet}$ \\
\hline & & & 400 & NMQ \\
\hline & & & 540 & $3^{+\bullet}, \mathrm{NMQ}^{\bullet}$ \\
\hline & & & $>700$ & $3_{2}^{+\bullet}$ \\
\hline & & & $325,530^{\mathrm{a}}$ & $3^{+\bullet}$ \\
\hline \multirow[t]{5}{*}{4} & $\mathrm{C}_{6} \mathrm{H}_{5}$ & $\mathrm{COOH}$ & $330-360$ & $4^{+\bullet} ; \mathrm{C}_{6} \mathrm{H}_{5} \mathrm{SC}\left(\mathrm{C}_{6} \mathrm{H}_{5}\right) \mathrm{H}^{\bullet}$ \\
\hline & & & 400 & NMQ \\
\hline & & & 520 & $4^{+\bullet}, \mathrm{NMQ}^{\bullet}$ \\
\hline & & & $>700$ & $4_{2}^{+\bullet}$ \\
\hline & & & $530^{\mathrm{a}}$ & $4^{+\bullet}$ \\
\hline
\end{tabular}




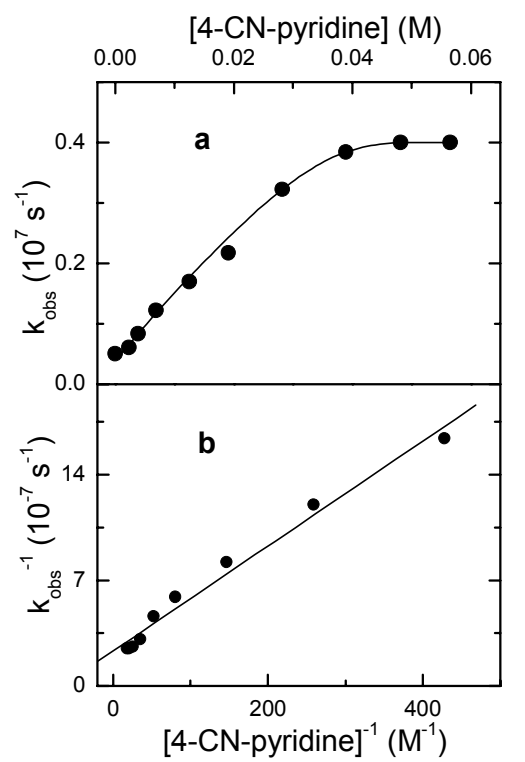

Figure S1. Observed rate constant ( $\left.k_{\mathrm{obs}}\right)$ vs [4-CN-pyridine] (a) and $1 / k_{\mathrm{obs}} v s 1 /[4-\mathrm{CN}$-pyridine] (b) for the decay of $\mathbf{3}^{+\bullet}$ in $\mathrm{CH}_{3} \mathrm{CN}$.

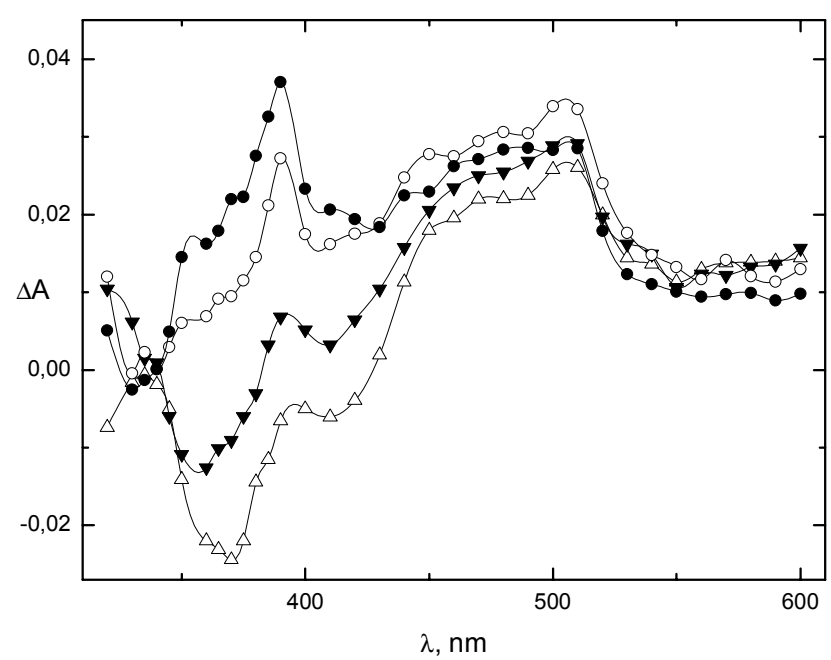

Figure S2. Time-resolved absorption spectra of the 1,4-dicyanonaphthalene $\left(1.0 \times 10^{-4} \mathrm{M}\right) /$

$\mathrm{PhSCH}(\mathrm{Ph}) \mathrm{CO}_{2} \mathrm{NMe}_{4}(0.01 \mathrm{M})$ system in $\mathrm{N}_{2}$-saturated $\mathrm{MeCN}$ recorded $0.14(\triangle), 0.18(\mathbf{\Delta}), 0.33($ o) and $3.2(\bullet) \mu$ s after the laser pulse. $\lambda_{\text {ecc }}=355 \mathrm{~nm}$. 